\title{
Medical Harm and Patient Safety-III: Procedural Errors
}

\author{
Prof. Neelam Adhikari \\ Rector, Patan Academy of Health Sciences, Lalitpur, Nepal
}

Address for correspondence: Dr. Neelam Adhikari, E-mail: neelamadhikari @gmail.com

Key words: Procedural errors, procedural and surgical site infections, protocols and guidelines, verification process, communication failure, teamwork

\section{Introduction}

$\mathrm{P}$ rocedural errors are failure to complete a procedure or surgery as planned or intended because of wrong plan to achieve the aim. These also include Post Procedural Errors (PPE). Whenever people sign off on consent forms that allow doctors to perform a procedure or surgery, they usually do not anticipate medical procedural errors to occur during these procedures. They usually assume that the physicians and surgeon has performed many such procedures and are therefore experienced and competent to actually perform this procedure once again without any error. Sadly this is not always true.

There is plenty of evidence that medical errors while performing an invasive procedure or during surgery occur with alarming frequency ${ }^{1,2}$. This is not often because people performing procedures or surgeons are incompetent or careless, but things go wrong at many steps involved in the procedure. Vincet and colleagues ${ }^{1}$ believed that procedural and surgical errors are associated with many other factors such as quality of design- interface, teamwork and organizational culture.

In addition health care associated infections can occur because of breaks in infection control protocols while performing the procedures or surgery. Inadequate communication- verbal and written plays very important role in causation of Procedural errors.

In the beginning I will give some examples of procedural errors that I encountered during my medical practice.

1. Performing pleural tap on the wrong site in a 5-year old child with right-sided pyothorax. The procedure was performed on left side and pronounced to be negative. This caused delay in relieving child's respiratory distress by institution of chest tube drainage, inability to get microbiological diagnosis and administration of appropriate antibiotics.

2. Two episodes of guide wires being left in while performing central venous catheterization in the intensive care unit, inadvertently. On one occasion mistake was recognized early and in another one realized late when patient took a bad turn and the guide wire was found on X-ray almost close to the heart. In both instances the patient had to be transferred to another hospital where guide wire was removed by catheterization procedure.

3. A sponge was left inadvertently inside the patient during a gynecological procedure.

4. Instances of many hospital acquired infections (because of break in infection control practices).

5. Delayed information of abnormal serum electrolyte report, where life-threatening hyperkalemia was present.

6. Delay in transfer of a severely dehydrated patient from emergency room to paediatric ward. The doctor wanted immediate transfer and institution of intravenous fluid administration to be started in the paediatric ward, as emergency room was busy. The transfer was delayed and intravenous fluids were not administered in emergency room.

7. Inadvertent discontinuation of oxygen while transferring a critically sick patient to Paediatric Intensive Care Unit. Even though discontinuation of oxygen was for a brief period, this resulted in severe emotional costs to the family, treating physicians and hospital management.

8. Inadequate follow of a critically sick patient by the night shift doctor because written handover failed to indicate the correct bed number and did not include patient's name. 
9. A 15 -year old boy sustained $60 \%$ body surface area burn. He was intubated and a central subclavian line was inserted, without x-ray confirmation. Over the next few hours, he became hypotensive, difficult to ventilate and hypoxic. The duty doctor attributed decreased pulmonary compliance to the burns. IV fluids and vasopressors were administered. The morning x-ray of the chest revealed a large pneumothorax. This was a PPE, where a routine of ordering the $\mathrm{x}$-ray after catheteriztion was missed.

Most of these errors are shocking. Due to the seriousness of that comes with procedural error consequences, the medical expenses in trying to fix these errors happen to be exorbitant. Nerve or organ damage can occur as well as scarring and infection. Victim's emotional toll also happens to be profound and several of them may never fully recover. Emotional toll on physicians is also profound; some of who may get clinically depressed or may even never work again ${ }^{3}$.

Post completion error (PCE) is a type of procedural error that occurs even when an individual has knowledge required to perform the task correctly. After performing a 'Task Critical Step' a PCE can occur if an individual forgets to perform a 'Related Step' before staring a new task ${ }^{4}$. These are akin to some common place examples of forgetting to remove the original after making a photocopy, leaving the card in the ATM after withdrawing the cash, and failing to replace the gas cap after filling up a car ${ }^{5}$. Likelihood of a PCE occurring could be predicted by the intrinsic difficulty associated with the main task, or the individual's ability to accurately remember information relevant to the task ${ }^{4}$. PCE can occur even if the individual is motivated or there is threat of consequences. It is more likely to occur when the record is not made immediately after completing the main task, instead the physician goes to perform other tasks and 'means' to do it later, increasing his own memory load.

The three main causes of adverse events in procedural and surgical care are ${ }^{3}$ -

1. Inadequate patient management.

2. Poor infection control methods.

3. Failure by health-care providers to communicate effectively before, during and after the procedure.

\section{Inadequate patient management}

When these events are analyzed, arrange of preexisting conditions (latent factors) have been identified. Some of these are:
1.1 Unavailability or inadequate implementation of protocols or guidelines.

1.2 Poor leadership.

1.3 Poor teamwork.

1.4 Conflict or lack of co-ordination between various departments.

1.5 Inadequate training and preparation of staff.

1.6 Inadequate resources.

1.7 Lack of evidenced-based practice.

1.8 Poor work culture.

1.9 Overwork.

1.10 Lack of system for managing performance.

Availability of protocols and guidelines, that also include post completion tasks, evidence-based resources close to working place, is a must. In this regard availability of evidence-based electronic resources such as Up-To-Date is a boon. Training of all staff, good role modeling, and reasonable duty rota, excellent handover practices cannot be over emphasized. Automation of even a commonly performed procedure should be cautious. The management must ensure adequate resources and have good system of evaluation of performance. Productive discussion at regular mortality and morbidity conferences is vital.

\section{Poor infection control practices}

The Harvard Medical Practice Study $\|^{2}$ found that surgical wound infections constituted the secondlargest category of adverse events. The implementation of safe infection control practices including appropriate antibiotic use throughout the hospital and appropriate administration of prophylactic antibiotics go a long way in preventing health care related infections. In addition increased attention to the risks of transmission healthcare workers how they as individuals and members of team can minimize risk of cross-infection.

Everyone has responsibility to decrease the opportunities for contamination of clothing, hands and equipment that have been associated with transmission routes. Role of infection control committee, availability of in-house infection control manual and training of all staff is vital.

\section{Failure by health-care providers to communicate effectively before, during and after procedures:}

One of the biggest problems during the procedure is miscommunication. Failures to communicate changes 
in the patient's condition have resulted in adverse events. Failing to communicate the perceived mistake of a 'senior' colleague is also miscommunication.

It has been reported that nurses are often silent about physician's errors. Almost $60 \%$ of nurses reported that they have experienced this kind of scenario, and $17 \%$ said it happened several times a month, according to a study by the American Association of Critical Care Nurses (AACCN), the Association of periOperative Registered Nurses (AORN), and a training company called VitalSmarts. This study aptly called "The Silent Treatment," physicians taking shortcuts, such as not washing their hands long enough, exhibiting incompetence, and demonstrating disrespect that shuts down lines of communication ${ }^{6}$. This study cites several anecdotes in which physicians blow off patient-safety protocols such as donning a full sterile barrier during arterial line placement. James Conway, a senior fellow at the Institute of Health $(\mathrm{IHI})$, said silent nurses have more to do with the wider issue of organizational culture ${ }^{7}$. "The vast majority of physicians want to do the right thing," he said. "They do not want their patients to have infections or stay in the hospital longer. But does the hospital give them time to wash their hands or put the soap dispenser in easy reach?" Another failure factor s leadership that pays only lip service to the new rules and procedures.

\section{Conclusion}

The causes of procedural errors are rooted at the deepest policy and organizational levels of medical care. These policies and organizational interactions are difficult to observe and measure. Errors can be minimized with diligent conversations with those who provide care $^{8}$.

Much is already known about how procedures can be safer. These strategies must be implemented in health care institutions.

\section{References}

1. Vincent C, Moorathy K, Sarkar SK, Chang A, Dorji AW. Systems approaches to surgical quality and safety: from concept to measurement. Ann Surg 2004;239(4):475-82.

2. Leape L, Troyan A. et al. The nature of adverse events in hospitalized patients: results of the Harvard Medical Practice Study II. N Engl J Med 1991; 323(6): 377-84.

3. Medical Malpractice. Medical Procedure Errors. Accessed; 2011 March 20. Available from: http:// www.malpracticemedical.co.uk/types-of-medicalmalpractice/medical-procedure-errors.html.

4. Jonathan Back, Wai Lok Cheng, Rob Dann, Paul Curzon and Ann Blandford. Does being motivated to avoid procedural errors influence their systamaticity? In Kinns NB, Blanford A, Curzon P, Nigay L editors. People and Computers $X X$-Engage Proceedings of $\mathrm{HCl}$. London: Springer-Verlag; 2007. P. 151.

5. Phillip H. Chung, Michel D. Byrne. Cue effectiveness in mitigating post completion errors in a routine procedural task. Inl J Human-Computer Studies 2008; 66(4):217-32.

6. Silent treatment. David Maxfield, Joseph Grenny, RamoALn Lavendrero and Linda Groah. In The silent treatment. Why safety tools and checklists aren't enough to save lives. 2005 Silence kills study. Accessed March 2011. Available from: www. silenttreatmentstudy.com

7. Medscape News. Robert Lowes. Nurses often silent about physician's mistakes. Accessed: March 2011. Available from: www.medscape.com/view article/739706.

8. McNutt RA, Abrams R, Arons DC; Patient Safety Committee. Patient safety efforts focus on medical errors. JAMA 2002; 287 (15): 1997-2001.

\section{How to cite this article?}

Adhikari N. Medical Harm and Patient Safety-III: Procedural Errors. J Nep Paedtr Soc 2011;31(2):157-159. 